\title{
Uso de Novas Tecnologias de Informação por Profissionais da Área da Saúde na Bahia
}

\author{
Gilberto Wildberger de Almeida \\ Ricardo Coutinho Mello
}

\begin{abstract}
Resumo
Uso de Novas Tecnologias de Informação por Profissionais da Área da Saúde na Bahia é um estudo descritivo sobre o grau de utilização da tecnologia da informação na área de saúde na cidade do Salvador, Bahia. O objetivo deste artigo é verificar a familiaridade que os médicos têm com a Tecnologia da Informação com vistas a se inferir procedimentos de relacionamento com os médicos e entre os principais atores da área da saúde. O estudo foi desenvolvido a partir de revisão de literatura e de múltiplas pesquisas de campo, realizadas entre médicos e usuários do segmento de laboratórios de análises clínicas. O levantamento realizado descreve o que é típico da relação que o médico tem com a tecnologia da informação e com os laboratórios de análises clínicas, bem como investiga as peculiaridades das relações existentes entre profissionais e pacientes, em que se revê o entendimento da prestação de serviços no setor. São utilizados percentuais, indicações de moda, medidas de posição e associação, por se tratar de estudo exploratório, com escalas nominais predominantemente. Os resultados demonstram que o uso intenso da tecnologia é uma tendência da população pesquisada e revela o grande impacto organizacional que pode ser proporcionado pela adoção de novas tecnologias no setor de saúde nos próximos anos.
\end{abstract}

Palavras-chave: tecnologia da informação; marketing de relacionamento; saúde.

\begin{abstract}
New Technologies of Information Use by Health Professionals in Bahia is a descriptive study of how much of the information technology is in usage amongst the health area in the city of Salvador, Bahia, Brazil. It was developed from the revision of literature and multiple field researches carried through medicine doctors and users of the segment of clinical analyses laboratories. The research describes what is typical in the relation that the doctor has with the information technology and with clinical analyses laboratories, as well as it investigates the peculiarities of the relationship of professionals with patients, reviewing the meaning of to provide assistance in the sector. Since it was an exploratory study with nominal scales, predominantly, it was used percentile indicators, mode, measures of position and association. The results demonstrate that the use of technology is a trend of the studied population and reveal that relevant impact is to be proportionated by the adoption of new technologies in the health sector in the following years.
\end{abstract}

Key words: information technology; relationship marketing; health. 


\section{INTRODUÇÃO}

Em nenhuma outra época histórica, a humanidade alcançou tantos avanços tecnológicos, em tão curto espaço de tempo, como no século XX. A transformação dos processos produtivos a partir da década de 50 revolucionou não apenas produtos e serviços, como também sociedades. Pressionadas pela macroestrutura competitiva internacional, coube às organizações repensar as estruturas de negócios para se adequar à nova era, visando maior participação de mercado e personalização de produtos e serviços. O objetivo deste artigo é verificar o impacto da tecnologia da informação numa área de serviços, o segmento da saúde, observando a atuação dos laboratórios de análises clínicas. Ademais, vamos analisar qual o grau de familiaridade que os médicos têm com a Tecnologia da Informação.

O atual estágio da tecnologia permitiu que a informação fosse tratada de forma eficaz, rápida e precisa, otimizando a complexa teia de dados sobre as preferências dos consumidores, em geral. O aumento considerável de dados à disposição dos gestores necessita de uma abordagem mercadológica para que decisões sejam tomadas eficazmente, a partir de informações relevantes, desconsiderando outras desprovidas de significado, para a construção de estudos sobre o consumidor e seus hábitos. Assim, as organizações em geral, incluindo as que atuam na área da saúde, devem revisar e ajustar suas práticas gerenciais para identificar e desenvolver estratégias que melhor se relacionem com todos os seus públicos. Entretanto o novo paradigma não reside somente no estabelecimento de estratégias para melhor conhecê-los, porque é crucial que as organizações saibam utilizar as ferramentas oriundas da informática com o fim de obter o melhor resultado no seu relacionamento com clientes diretos e indiretos.

Os serviços não apenas precisam ser ajustados à eficácia gerencial dos processos, como devem estar comprometidos com a inovação constante, abrindo novas frentes de trabalho, a partir da área mestra da organização (Hamel e Prahalad, 1995). Todavia a inovação extrapola a simples automação de processos administrativos. Sem projeto de longo prazo para o uso da tecnologia na organização, o processo de criação de valor para o consumidor é ineficaz, revelando um desconhecimento da função instrumental da informática por parte da organização (Canesqui et al., 2000). Outra prática muito usual é a perda de foco no cliente, 
centrando a estratégia somente na tecnologia incorporada (orientação para o produto).

O conceito de Customer Relationship Management (CRM), Gerenciamento das Relações com o Consumidor, surge como desdobramento evolutivo dos sistemas de gestão, passível de uso na área da saúde, em particular dos módulos de Enterprise Resource Planning (ERP), Planejamento de Recursos Corporativos, tais como materiais, contas a pagar e a receber, entre outros. A migração de dados de um sistema para outro ainda se tem revelado pouco prática, dada a utilização distinta e o teor das informações processadas. Enquanto o CRM deve ser percebido como o ponto de partida para o delineamento de estratégias personalizadas para grande gama de consumidores (Peppers e Rogers, 2000), os programas de ERP servem ao propósito de consagrar os princípios de acompanhamento e controle contábil no segmento da saúde.

No atual momento, somente as organizações de grande porte podem arcar com o investimento requerido, para que se viabilize a propagação do CRM na estrutura organizacional, ou seja, a incorporação, análise e gestão da base de dados de sua clientela. Porém, de acordo com projeções, este custo deve ser reduzido no curto prazo, considerando o desenvolvimento de novas tecnologias que permitirão à técnica tornar-se instrumento de suporte da comercialização para variados tipos de organizações, incluindo as de serviço. Nosso interesse, neste artigo, é verificar até que ponto os médicos estariam dispostos a adotar essas ferramentas contemporâneas.

Em segunda instância, de posse das informações geradas, e com base nas relações de atendimento pré-existentes, vislumbra-se que os atores escolhidos como representantes do segmento da saúde possam identificar perfis de conduta e preferência, agrupando seus clientes em comunidades virtuais de relacionamento. Criada a partir de banco de dados, a microssegmentação em comunidades facilita a aplicação de práticas comerciais interativas que atendam por completo as necessidades específicas dos consumidores, assegurando, assim, não apenas a fidelidade ao médico. O conceito de inteligência de negócio nos serviços prestados na área de saúde pode ser enfocado como exemplo que vai mais além do viés técnico-comercial, da gestão de preferências e necessidades do cliente. Numa sociedade onde comportamentos e valores estão em contínua e acelerada transformação, a tecnologia torna-se instrumento básico das organizações no planejamento, desenvolvimento e adoção de ajustes sistemáticos na estratégia empresarial de forma rápida e instantânea para obter, desta forma, vantagens competitivas em relação à concorrência. 
As peculiaridades da relação médico-paciente, típicas da área de saúde, forçam uma revisão completa no entendimento da prestação de serviços; e este é o problema. A prática médica insere-se num emaranhado conjunto de princípios éticos e sociais que dificulta a definição cartesiana de consumidor, fornecedor e prestador de serviços. Quando se avaliar a atuação dos laboratórios de análises clínicas, identificam-se, inicialmente, dois tipos de clientelas: a clientela médica e a do consumidor final, o paciente.

Os avanços tecnológicos trouxeram melhorias para a prática médica. Entretanto as empresas da área de saúde têm dificuldade em proporcionar melhorias à clientela, seja na prestação dos serviços, seja na divulgação destes. Pretende-se analisar em que medida o investimento em novas tecnologias de informação otimiza a qualidade dos serviços prestados a diversas categorias de cliente, e como os dados gerados por esses sistemas estão atrelados a um macroprocesso de relacionamento interpessoal mantido pela organização, ou sendo utilizado para se criar uma cultura que favoreça o contínuo aprendizado sobre as preferências do consumidor.

Como intermediadora de ações e de informações entre duas clientelas distintas, uma organização que presta serviços laboratoriais pode contar com a tecnologia para elaborar estratégias de relações públicas, unindo virtualmente consumidores, a partir do processamento de informações. A criação e manutenção de comunidades virtuais é um aspecto que pode resultar do uso adequado da tecnologia por médicos, sobretudo em face da preocupação ética de se transmitir aos clientes notícias periódicas, de modo personalizado, sem ferir a intimidade dos envolvidos, mas ao mesmo tempo estimulando a participação dos dois públicos privilegiados. Um exemplo de comunidade de consumidores seria a organização de grupos de diabéticos, ou de pessoas portadoras de esclerose múltipla. Assim, pessoas com a mesma doença podem ser coordenadas por profissionais de saúde a trocar experiências, apoiando-se mutuamente e permitindo que ocorra a possibilidade de aprendizado coletivo.

\section{Especificidade dos Serviços de Saúde}

Uma das dificuldades de se estudar o segmento de serviços são as características deste ramo de atividade econômica. Dada a intangibilidade e a imaterialidade dos serviços (Téboul, 1999), a localização de uma organização 
tem importância singular na definição da estrutura de negócio (Kotler, 1991). A localização transmite, subliminarmente, à clientela conceitos como: competência ou expertise dos funcionários; precisão, cortesia, rapidez, receptividade e criatividade da equipe; segurança e reconhecimento das necessidades do cliente (Kotler, 1991). São também características comumente relacionadas aos serviços as seguintes:

(i) inseparabilidade, i. e. os serviços são prestados e consumidos no mesmo momento;

(ii) variabilidade, i. e. dependência da habilidade do prestador do serviço, e;

(iii) perecibilidade, i. e. os serviços não podem ser estocados.

Porém Castells (1999) indica que no paradigma informacional tais características esmaecem em decorrência da interpenetrabilidade do conceito de serviços e produtos, assim como ocorre com os setores da economia.

É relevante considerar, sem embargo, a distinção que Téboul (1999) estabelece para os serviços quanto à assistência do consumidor, caracterizando-os como serviços de linha de frente (front-office), e de bastidores (back-office). Na primeira tipologia, encontram-se as principais estratégias que manipulam as clássicas funções do marketing e estão mais orientadas para o cliente, requerendo personalização, flexibilidade, menos especializações e mais envolvimento com o público externo.

Os serviços de bastidores, por outro lado, são mais orientados ao produto. Com a Era da Informação, as contribuições da tecnologia têm favorecido igualmente a ambos, permitindo que as organizações apurem o valor do relacionamento com a clientela, privilegiando o uso intensivo do instrumental típico de Relações Públicas. Com efeito, nos dias que correm, as funções de Relações Públicas ficam cada vez mais próximas dos profissionais de Marketing em função sobretudo, da Tecnologia da Informação.

Téboul (1999) cita os elementos essenciais que precisam ser observados para a prestação de serviços com valor agregado à clientela: resultados, processo, funcionários, confiabilidade e preço. Dentro deste espectro, em todas as dimensões que permeiam a interação com a clientela, o contínuo aprendizado das necessidades e preferências da clientela deve estar sempre em foco. Igualmente, sutis variáveis devem ser valorizadas pela organização, interna e externamente à estrutura.

Quanto ao poder da influência da tecnologia sobre a prática do setor de serviços 
deve-se considerar que há singularidades. Para mensurar e identificar o fluxo de dados, a sociedade tende a materializar a onipresença da informação, centrando esforços em aparatos tecnológicos cada vez mais avançados. Os serviços prestados pelos profissionais e organizações privadas devem acompanhar o novo padrão tecnossocial vigente (Andreazzi, 2000). Visando à competitividade, as organizações são compelidas a revisar a teia das relações estabelecidas com clientes e fornecedores, recorrendo ao uso intensivo da tecnologia. O advento de práticas profissionais, fortemente alicerçadas em tecnologias de informação, como a telemedicina, é um caso ilustrativo. No afã de diferenciação, as organizações exibem equipamentos de ponta sofisticados, para atrair a atenção da sociedade. No entanto, na maior parte das organizações do setor de saúde no Brasil, o uso da tecnologia da informação ainda está associado ao mero suporte das atividades de controle e prestação de contas, visando a atender requerimentos de cunho fiscal.

\section{Serviços de Saúde no Brasil}

Na década de 50 , as mudanças macroeconômicas proporcionaram o investimento estrangeiro no Brasil e, por extensão, a reorganização da cadeia produtiva e dos serviços em geral. Com o aumento de informação disponível, as organizações e os profissionais foram compelidos a prestar serviços de melhor qualidade que atendessem às necessidades do consumidor, aferindo indicadores de produtividade e lucratividade (Sant'anna, 1998).

Na área de saúde não foi diferente. A demanda por acesso a serviços de melhor qualidade obrigou a reorganização dos processos de trabalho, das relações dos profissionais de saúde com a população e do emprego de novas tecnologias. Apesar de ser um setor altamente regulamentado por entidades de classe e autoridades governamentais, os profissionais de saúde tiveram que se adaptar à Era da Informação.

Segundo Canesqui et al. (2000):

“(...) os avanços científicos e tecnológicos ampliaram o conhecimento e as possibilidades de diagnósticos, tratamentos e prognósticos das doenças, tornando-se difícil imaginar um profissional que dê conta de todo esse universo" Canesqui et al. (2000, p. 108). 
A prática médica concentrou-se na medicina tecnológica, com subseqüente e intermitente divisão do trabalho, orbitando os avanços em torno do eixo de grandes instituições de saúde e do surgimento de muitas subespecialidades médicas, principalmente voltadas para o uso da imagem nas tecnologias bioquímicas de diagnóstico-terapia. Neste espectro, encontram-se os profissionais com funções exclusivamente analíticas e descritivas, como é o caso dos médicos patologistas, radiologistas e dos farmacêuticos bioquímicos.

De acordo com Canesqui et al. (2000), as especialidades médicas podem ser divididas em três agrupamentos sociológicos, no que concerne ao uso da tecnologia instrumental e ao envolvimento do profissional com o paciente (Quadro 1).

\section{Quadro 1: Tipos de Especialidades Médicas por Grupo Sociológico}

\begin{tabular}{|c|l|l|}
\hline Envolvimento Tecnológico & \multicolumn{1}{|c|}{ Categoria } & \multicolumn{1}{c|}{ Especialidades } \\
\hline Alto & Cognitivas & $\begin{array}{l}\text { Clínica médica } \\
\text { Pediatria }\end{array}$ \\
\hline Médio & Intermediárias & $\begin{array}{l}\text { Cardiologia } \\
\text { Gastroenterologia }\end{array}$ \\
\hline Baixo & Técnicas ou de & $\begin{array}{l}\text { Especialidades Cirúrgicas } \\
\text { Patologia } \\
\text { Radiologia } \\
\text { Bioquímica }\end{array}$ \\
\hline
\end{tabular}

Adaptado de Canesqui et al. (2000).

Uma característica do setor de saúde, além da padronização dos serviços, é a difícil distinção entre os públicos, dado o alto grau de dependência e inter-relação que há do profissional para o cliente. Historicamente, a prática médica tem influenciado o imaginário da comunidade. O profissional médico é ora percebido como mago, ora percebido dentro da mais alta estima pela sociedade (Canesqui et al., 2000). Entretanto, para o paciente, a competência do profissional de saúde está condicionada ao conhecimento e à prescrição dos mais modernos exames diagnósticos, relegando a uma instância secundária os tradicionais procedimentos para identificar patologias:

“(...) o reconhecimento público de um bom profissional de saúde passa não somente por sua capacidade de adquirir equipamentos médico- 
hospitalares de última geração, mas também pela freqüência com que solicita a realização de exames laboratoriais e prescreve a utilização de medicamentos” (Canesqui et al., 2000, p.158).

Entrementes, a introdução de novas tecnologias representa um ônus a mais para o paciente, reforçando o caráter hegemônico do acesso à saúde. De acordo com os autores, o desejo do profissional médico é não agregar tecnologia unicamente, mas obter precisão e rapidez nos resultados, gerando o mínimo de desconforto para o paciente, o que possibilita a adesão imediata à terapêutica. Em um senso estratégico, o pronto compromisso assumido pelo paciente com o tratamento restringe a busca por uma segunda opinião, cerceando a forte concorrência que o médico sofre.

Canesqui et al. (2000) levantam pontos críticos nas condições de trabalho do cotidiano dos profissionais médicos. No decorrer desta análise, serão destacadas algumas que implicam em maior constrangimento estratégico da prática gerencial, a saber:

(i) Aumento de custos da produção e preços para consumo.

(ii) Dependência de tecnologias e de suas indústrias.

(iii) Perda da carteira de clientes das organizações para as gerenciadoras de plano de saúde.

(iv) Heterogeneidade da relação médico-paciente.

Todas essas considerações comentadas foram arroladas como passíveis de serem aproveitadas em trabalho que visa a estudar a estreita inter-relação do setor de serviços de saúde com o uso da tecnologia, como elemento estratégico na prática gerencial. Convém salientar que outro reflexo da abertura da economia foi o incremento na taxa de incorporação de novas tecnologias para equipamentos e insumos. Na área de saúde, diferentemente de outros setores, a adoção de avanços tecnológicos como suporte de diagnóstico, abriu o caminho para a adoção da Tecnologia da Informação por parte dos principais atores.

\section{Os Médicos e a Tecnologia da Informação}

Segundo Maia Filho (2000), o mercado de saúde no Estado da Bahia movimenta 
cerca de US\$ 937,5 milhões por ano. A participação de negócios que envolvem a iniciativa privada é de, aproximadamente, 53\% deste montante. A área de saúde é a responsável pela segunda maior arrecadação do Imposto Sobre Serviços (ISS) do município de Salvador: 45 mil profissionais, cerca de $5 \%$ da população economicamente ativa, trabalham na área de saúde.

Estima-se que 34 milhões de pessoas são atendidas por planos privados de saúde em todo o Brasil, representando $25 \%$ da população. No Estado da Bahia, a média de usuários é inferior à nacional, 14\%, correspondendo a 1,8 milhão de pessoas. Os gastos anuais dos usuários com mensalidade giram em torno de US\$ 495 milhões. Na Bahia, há 45 operadoras interagindo com 1800 prestadores de serviços privados, dos quais $60 \%$ se encontram na capital.

Cabe, pois, concluir que os planos de saúde têm forte influência e representatividade na área de saúde. Para aferir a sua produtividade, as grandes operadoras de planos de saúde, geridas, na maior parte das vezes, por grupos internacionais e/ou instituições financeiras, ampliaram a análise de valor da cadeia produtiva para a gestão de negócio dos agentes do setor. Introduziram-se prérequisitos de atuação em um mercado globalizado para os empresários e prestadores de serviços de saúde. Como novidade, surge um elemento para modificar os parâmetros da assistência de saúde: o auditor das contas médicas.

A busca de redução de custos, materializada na figura do auditor dos planos de saúde, contribui para que a gestão dos negócios esteja, cada vez mais, centrada na ótima gestão de recursos. Ocorre, então, um deslocamento de atenção do paciente para o custo do atendimento. A visão que os empresários e profissionais do setor têm a respeito desta nova variável é a mais nefasta. Por meio do auditor, o caráter economicista é reforçado na prática médica. Este fato, aliado a tendência no segmento médico em tratar o paciente de forma fragmentada, ou seja, privilegiando a patologia e a diagnóstico-terapia em detrimento de uma abordagem individualizada na assistência, repercute negativamente na qualidade dos serviços prestados.

Contrapondo-se à corrente de redução de custos, detecta-se uma demanda crescente da clientela, principalmente a usuária de planos de saúde, por melhor qualidade na prestação de serviços. A qualidade, no entanto, é dependente direta de investimentos na estrutura de negócio, tais como capacitação de pessoal, certificação ISO 9002, investimento em novos aparelhos, entre outros.

No caso dos laboratórios de análises clínicas, uma das premissas para a lucratividade é que a classe médica deva estar fortemente envolvida na estrutura 
de negócio, porque, segundo a pesquisa da FIOCRUZ, 79\% dos médicos no Brasil atuam em parceria com convênios de saúde. Com o objetivo de atrair e cativar médicos, principalmente os que detêm altos índices de encaminhamento de pacientes, laboratórios de análises clinicas tendem a investir em marketing de relacionamento, por meio até mesmo de comunidades informacionais, conforme pesquisa dos autores, conduzida em Salvador, para buscar dados e informações que pudessem orientar o fio condutor deste trabalho.

Portanto, com o objetivo de avaliar como o médico usufrui toda a potencialidade dos avanços tecnológicos, aplicou-se uma pesquisa probabilística aleatória simples, no período de novembro a dezembro de 2000, tendo como universo os médicos do Estado da Bahia. Com este tipo de amostragem, assegurou-se a possibilidade igual de participação dos médicos na pesquisa. Um pressuposto, a ser confirmado, é que os indivíduos nascidos a partir de 1950 têm maior propensão e facilidade em utilizar a tecnologia corriqueiramente, uma vez que a prática médica, antes da Era da Informação, dependia muito da habilidade do profissional em se relacionar com pacientes, colhendo e analisando dados para proporem terapêuticas. Como a escalada das inovações tecnológicas foi mais intensa a partir da década de 70 , supõe-se que a formação dos profissionais egressos da universidade neste período tenha sido mais voltada para os sistemas tecnológicos, diferentemente dos seus predecessores, que desenvolviam mais o fator humano na assistência.

A pesquisa realizada descreve a relação que o médico tem com a mais básica e simples manifestação da tecnologia da informação: o uso de Internet e de telefones celulares entre os médicos. A opção por um estudo com amostragem aleatória foi em função do tempo e dos recursos disponíveis. Todavia reitera-se que a população é representativa do universo, por ter sido utilizada uma amostragem metodológica que oferece chances iguais de participação aos indivíduos do universo pesquisado.

As medidas de posição utilizadas são os percentuais e indicação da moda, por se tratar de estudo exploratório, com escalas nominais predominantemente. Em alguns dados, são usadas medidas de associação para se avaliar o nível de relacionamento entre variáveis. Algumas perguntas foram lançadas em duplicidade no questionário com o intuito de se confirmar padrões. Aos médicos foi perguntado também qual a avaliação deles sobre os laboratórios de análises clínicas. O objetivo das questões sobre os laboratórios foi verificar se o uso das novas tecnologias de comunicação e informação - sem considerar equipamentos técnicos usados nos procedimentos de análise laboratorial - e seu uso no relacionamento de informação com os clientes e para os clientes -tanto médicos como pacientes - influi no conceito que os médicos fazem sobre os laboratórios. 


\section{A Amostragem}

De acordo com o Conselho Regional de Medicina do Estado da Bahia (CREMEB), em torno de 500 profissionais registram-se anualmente para obter o direito de exercer a profissão no Estado da Bahia. Entre eles, encontram-se os recém-formados e profissionais transferidos de outras regiões do país. Estimase que estes últimos representem em torno de $10 \%$ do universo de novos registros, uma vez que na Bahia as duas únicas faculdades de medicina lançam no mercado cerca de 360 profissionais por ano. Como o registro no CREMEB é obrigatório para o exercício da profissão, recorreu-se à estratificação do universo em duas classes. A primeira compreende os registros abaixo de 6000, e a segunda, os restantes. Estabeleceu-se o registro 6000 como referencial para a população da amostra, porquanto, em torno deste encontram-se os médicos que obtiveram graduação entre 1974 e 1980 e, supostamente, nascidos entre 1950 e 1956. A idade aproximada de um médico para a conclusão do curso de graduação na Bahia é de 25 anos, sem considerar o período de residência. Ao examinar a lista do CREMEB, verifica-se que cerca de 76\% dos profissionais estão com registros ativos na Instituição, percentual muito superior ao encontrado nacionalmente pela pesquisa da FIOCRUZ (66,7\%). Dos ativos no CREMEB, aproximadamente, $60 \%$ estão acima do número 6000. A distribuição de registros no total de ativos é comparada com a encontrada em cada classe no Quadro 2.

Quadro 2: Resumo Estatístico dos Registros no CREMEB

\begin{tabular}{l|r|r|r} 
Registros & $\begin{array}{c}\text { Total de } \\
\text { Ativos }\end{array}$ & $\mathbf{< 6 0 0 0}$ & \multicolumn{1}{l}{$\mathbf{6 0 0 0}$} \\
\hline Média & 7.719 & 3.514 & 10.228 \\
Mediana & 7.691 & 3.616 & 10.233 \\
Intervalo & 14.368 & 5.993 & 8.370 \\
Menor Registro & 3 & 3 & 6.001 \\
Maior Registro & 14.371 & 5.996 & 14.371 \\
Contagem & 10.908 & 4.076 & 6.832 \\
\hline
\end{tabular}

Fonte: baseado na listagem do CREMEB, dez. 2000. 
Estabelecendo um grau de confiança de 95\%, um erro amostral de 8\% e uma participação de $60 \%$ para os médicos com o registro acima de 6000 , obtém-se uma amostra de 150 indivíduos. O modelo estatístico, referendado para o cálculo da amostra, é o apresentado por Tagliacarne (1978):

\section{FÓRMULA para Cálculo de Amostragem}

$$
\begin{aligned}
& n=\frac{4 \times p \times q}{E^{2}}, \text { onde }: \\
& \mathrm{n}=\text { amostra } \\
& \mathrm{p}=\text { porcentagem de ocorrência do fenômeno } \\
& \mathrm{q}=\text { porcentagem complementar }(100-\mathrm{p}) \\
& \mathrm{E}=\text { erro amostral }
\end{aligned}
$$

Da lista do CREMEB foram escolhidos aleatoriamente 170 médicos. Os sorteios foram efetuados conforme a proporção estabelecida para o cálculo da amostra. Os questionários foram aplicados em campo, por meio da visita aos profissionais na capital, sendo recolhidos dez dias depois, e enviados pelo correio aos localizados no interior da Bahia.

Em geral, o profissional médico é de difícil acesso e pouco propenso a colaborar com esse tipo de investigação. Porém a incidência de erro não amostral do tipo não-resposta foi baixa (2\%), sendo descartados os questionários que excederam o tamanho da amostra definido previamente. Atribui-se ao baixo índice de não-resposta o contato reiterado com os entrevistados após o sorteio, para enfatizar a importância em participar do estudo e observar o prazo para a entrega do questionário.

\section{Análise dos Resultados}

As especialidades mais freqüentes na amostra obtida são a ginecologia, pediatria e a clínica médica (Quadro 3). A distribuição por gênero é equilibrada, com discreta prevalência de mulheres (51\%) sobre os homens, confirmando as predições de Canesqui et al. (2000) sobre a tendência do predomínio do sexo feminino nas profissões de saúde. 


\section{Quadro 3: Freqüência de Especialidades Médicas na Pesquisa}

\begin{tabular}{l|r|r}
\hline Especialidades & Casos & \% \\
\hline Anestesiologia & 3 & 2,0 \\
Cardiologia & 3 & 2,0 \\
Cirurgia & 17 & 11,3 \\
Clínica Médica & 21 & 14,0 \\
Dermatologia & 6 & 4,0 \\
Gastroenterologia & 3 & 2,0 \\
Ginecologia & 39 & 26,0 \\
Infectologia & 1 & 0,7 \\
Mastologia & 2 & 1,3 \\
Nefrologia & 2 & 1,3 \\
Neurologia & 2 & 1,3 \\
Oftalmologia & 4 & 2,7 \\
Oncologia & 2 & 1,3 \\
Ortopedia & 4 & 2,7 \\
Otorrinolaringologia & 3 & 2,0 \\
Pediatria & 21 & 14,0 \\
Pneumologia & 8 & 5,3 \\
Psiquiatria & 2 & 1,3 \\
Reumatologia & 2 & 1,3 \\
Urologia & 3 & 2,0 \\
UTI & 2 & 1,3 \\
População da Amostra & $\mathbf{1 5 0}$ & $\mathbf{1 0 0 \%}$ \\
\hline
\end{tabular}

Nota: algumas sub-especialidades foram agrupadas por área mais representativa.

No que concerne aos meios de comunicação utilizados, observa-se uma elevada adesão dos profissionais ao telefone celular (Quadro 4), principalmente entre os profissionais mais jovens (Quadro 5), o que confirma a expectativa inicial deste estudo. Como múltiplas respostas podem ocorrer no quesito que trata desse assunto, o e-mail é também citado pelos respondentes, mas em menor proporção. 


\section{Quadro 4: Meios de Comunicação que Auxiliam o Médico}

\begin{tabular}{l|r|r} 
Aparelhos / Serviços & Casos & \multicolumn{1}{|c}{$\%$} \\
\hline Fax & 71 & 47,3 \\
Telefone Celular & 130 & 86,7 \\
E-mail & 82 & 54,7 \\
Pager ("Bip") & 14 & 9,3 \\
Outros & 42 & 28,0 \\
Nenhum & 2 & 1,3 \\
\hline
\end{tabular}

\section{Quadro 5: Registro no CREMEB vs. Uso de Telefone Celular}

\begin{tabular}{l|r|r|r}
\multirow{2}{*}{ Reg. no CREMEB } & \multicolumn{3}{|c}{ Telefone Celular contribui? } \\
\cline { 2 - 4 } & \multicolumn{1}{|c|}{ Não } & \multicolumn{1}{|c}{ Sim } & \multicolumn{1}{c}{ Total } \\
\hline Abaixo de 6000 & 16 & 44 & 60 \\
Acima de 6000 & 4 & 86 & 90 \\
Total de Casos & $\mathbf{2 0}$ & $\mathbf{1 3 0}$ & $\mathbf{1 5 0}$ \\
\hline
\end{tabular}

Na questão que trata das fontes de atualização dos médicos, já eram esperadas como respostas mais freqüentes as relacionadas à participação em eventos e atualização por meio de publicações médicas (Quadro 6). Surpreendente, no entanto, é o percentual de indicação da Internet como fonte de consulta. Alguns laboratórios de análises clínicas, por exemplo, estimam que 30\% dos médicos com que se relacionam tem acesso à Internet. Se comparado o percentual aos resultados da população da amostra, conclui-se que a percepção pode estar subestimada, pois cerca de $55 \%$ dos médicos afirmam utilizar o e-mail corriqueiramente e $70 \%$ consideram a Internet como fonte de atualização profissional. 


\section{Quadro 6: Fontes de Atualização Profissional}

\begin{tabular}{l|r|r}
\hline Meios de Informação & \multicolumn{1}{|c|}{ Casos } & \multicolumn{1}{l}{$\%$} \\
\hline Eventos & 132 & 88,0 \\
Publicações Médicas & 140 & 93,3 \\
Outras Fontes & 43 & 28,7 \\
Internet & 105 & 70,0 \\
Outras Publicações & 38 & 25,3 \\
Nenhum & 1 & 0,7 \\
\hline
\end{tabular}

Outra possibilidade é que o percentual citado pelo ALPHA se refira a um grupo de médicos, onde há predomínio dos que têm registros no CREMEB abaixo do número 6000, como se verifica na pesquisa.

Quadro 7: Registro vs. Uso da Internet

\begin{tabular}{l|r|r|r}
\hline \multirow{2}{*}{ Reg. no CREMEB } & \multicolumn{3}{|c}{ Usa Internet? } \\
\cline { 2 - 4 } & \multicolumn{1}{|c|}{ Não } & \multicolumn{1}{c|}{ Sim } & \multicolumn{1}{c}{ Total } \\
\hline Abaixo de 6000 & 24 & 36 & 60 \\
Acima de 6000 & 21 & 69 & 90 \\
Total de Casos & $\mathbf{4 5}$ & $\mathbf{1 0 5}$ & $\mathbf{1 5 0}$ \\
\hline
\end{tabular}

\section{Gráfico 1: Registro no CREMEB vs. Uso da Internet}

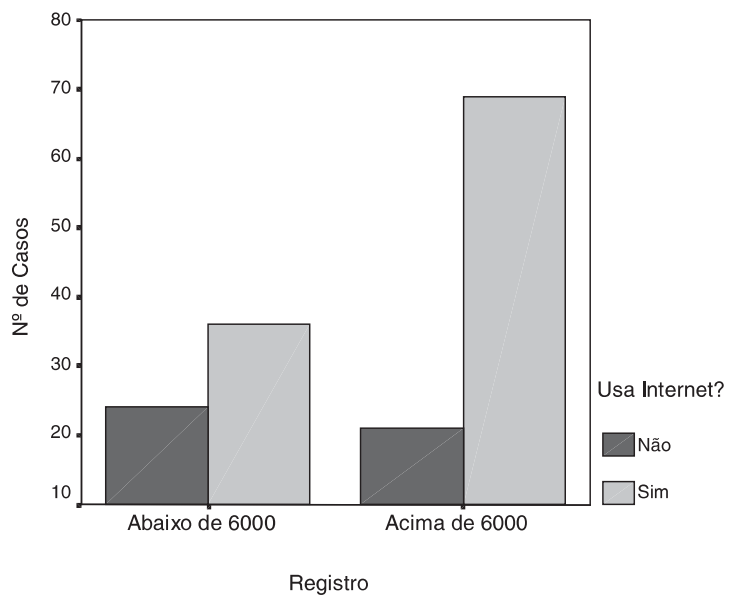




\section{Quadro 8: Registro no CREMEB vs. Contribuição do E-mail para a Profissão}

\begin{tabular}{l|r|r|r}
\multirow{2}{*}{ Reg. no CREMEB } & \multicolumn{3}{|c}{ E-mail contribui? } \\
\cline { 2 - 4 } & \multicolumn{1}{|c|}{ Não } & \multicolumn{1}{|c|}{ Sim } & Total \\
\hline Abaixo de 6000 & 29 & 31 & 60 \\
Acima de 6000 & 39 & 51 & 90 \\
Total de Casos & $\mathbf{6 8}$ & $\mathbf{8 2}$ & $\mathbf{1 5 0}$ \\
\hline
\end{tabular}

Detecta-se na amostra uma não proporcionalidade no uso de duas tecnologias interligadas. Uma possível explicação para o fenômeno é que os médicos utilizam a Internet, por meio da World Wide Web, para fins educacionais e de entretenimento, enquanto o $e$-mail é destinado à troca de informações de cunho técnico.

Com o objetivo de avaliar o grau de associação entre variáveis pertinentes ao problema, aplicou-se um teste estatístico, o Coeficiente de Contingência. O grau encontrado de associação do uso de $e$-mail com a participação em eventos foi de 23\%. Quando comparado o uso da Internet com as publicações médicas como fontes de atualização, obteve-se um coeficiente de 17\%, na ordem de 3\%. Cabe informar que a principal fonte de consulta utilizada pela classe médica para atualização científica é o MEDLINE, acervo bibliográfico que reúne as principais publicações em todo o mundo, disponível no ambiente web.

Ratifica-se, portanto, a importância da avaliação do perfil da clientela-chave, por meio de técnicas comerciais que empregam largamente a tecnologia, como o CRM, pela organização para melhorar o diálogo com os públicos. Utilizando canais de comunicação adequados ao perfil do cliente que se pretende fidelizar, as práticas comerciais tendem a ser mais vantajosa para todas as partes.

Todavia é prática pouco comum do segmento de análises clínicas servir-se de outro tipo de tecnologia que não a instrumental no dia-a-dia. Afora um projeto vanguardista, declarado por um laboratório de Salvador, não há histórico do uso da tecnologia da informação como instrumento corriqueiro para alavancagem comercial. A tênue corrente identificada por esse laboratório favorável ao uso intensivo da tecnologia da informação como base para construção de relações comerciais, em curto prazo, pode ser meramente uma lívida intenção desse laboratório em se alinhar ao novo paradigma sem, no entanto, imergir pronta e integralmente toda a estrutura de negócios em novas práticas comunicacionais intensivas em tecnologia. 
Na pesquisa fica evidente que, dos médicos que responderam favoravelmente à participação em uma comunidade informacional, a maioria já faz uso da Internet; portanto conhece os benefícios e problemas atuais da grande rede, ainda que superficialmente. A razão entre a rejeição da comunidade informacional e a sua adesão a ela (1:3) é a mesma dentro das classes (Quadro 9). Em síntese, a comunidade informacional aparenta despertar, pelo menos, a curiosidade da população estudada. Sem embargo, cumpre destacar que alguns médicos fizeram anotações no questionário, indicando a preferência por somente uma das proposições, e-mail ou celular, mesmo sendo estas indissociáveis, dada a existência de um operador lógico (ou) na formulação da questão.

\section{Quadro 9: Registro no CREMEB vs. Participação em Comunidade Informacional}

\begin{tabular}{l|r|r|rr}
\hline \multirow{2}{*}{ Reg. no CREMEB } & \multicolumn{3}{|c}{ Participar de comunidade? } \\
\cline { 2 - 4 } & Não & \multicolumn{1}{|c|}{ Sim } & Total \\
\hline Abaixo de 6000 & 15 & 45 & 60 \\
Acima de 6000 & 22 & 68 & 90 \\
Total de Casos & 37 & $\mathbf{1 1 3}$ & $\mathbf{1 5 0}$ \\
\hline
\end{tabular}

A proposta do laboratório, citado acima como vanguardista, no que diz respeito à comunidade informacional, é agregar pacientes com segmentação socioeconômica semelhante para encaminhar boletins informativos por meio de telefones celulares e de $e$-mail. Não obstante, para que a reunião de médicos em grupos favoreça a relação da organização com a clientela, é importante que os usuários percebam o valor da comunidade (Shapiro e Varian, 1999) e também sejam ativos na troca de informações entre si. Com efeito, a chave de sucesso para a inovação é a rapidez e a simplicidade de serviços com alto valor agregado. O serviço de entrega de laudos, por exemplo, que leva cerca de uma hora para chegar ao paciente e deste ao médico, poderá estar disponível para o profissional tão logo seja efetuado o processamento do exame. O próprio equipamento que processa o material biológico transmitirá o resultado. Com o sistema $M 2 M$, esperase que os resultados dos exames sejam enviados para o médico quase em tempo real, uma vez que o tempo de transmissão levará menos de um minuto.

Para uma organização, a tecnologia pode ser a fonte de soluções e, ao mesmo tempo, foco de sistemas informativos ineficazes, porquanto, a despeito de todo o avanço tecnológico na ciência da computação, são comuns falhas resultantes tanto de aspectos técnicos, de hardware, como de aspectos ambientais, o que 
inclui o fator humano. A fim de que a tecnologia seja aplicada de forma profícua pela organização, múltiplos fatores interdependentes precisam estar ajustados e sincronizados. É obrigatório que a sociedade e o espaço organizacional estejam aptos a acolher a tecnologia sem contenções de insumos. Um exemplo paradoxal é o sistema bancário. A despeito do alto grau de complexidade dos sistemas informativos, os serviços prestados castigam o usuário com freqüentes interrupções no atendimento, por causa da baixa qualidade da rede elétrica e de telecomunicações como um todo, no país. É o risco que corre um laboratório, ao dirigir a estratégia do negócio para um padrão tecnológico muito superior ao encontrado na sociedade.

Em suma, à guisa de conclusão, observa-se que o uso intenso da tecnologia é tendência da população pesquisada. Assume-se como fator decisivo para o incremento do uso da tecnologia o rejuvenescimento do efetivo de médicos com registro no conselho da categoria. As gerações mais antigas tendem a ser substituídas por novos profissionais, cuja formação profissional privilegia a tecnologia como apoio à prática médica, o que demonstra a validade de se construir uma comunidade informacional. Num amplo espectro de potencialidades a serem exploradas, entende-se a constituição deste tipo de comunidade como forma avançada de marketing de relacionamento, proporcionando benefícios para todos os públicos: médicos, pacientes e laboratório.

\section{Artigo recebido em 10.06.2003. Aprovado em 19.12.2003.}

\section{REFERÊNCIAS}

ANDREAZZI, M. et al.

O Setor de Saúde na Perspectiva da Dinâmica da Inovação. In: CONGRESSOBRASILEIRO DE SAÚDE COLETIVA, 6., 2000, Salvador. Anais... v.5. Salvador:ABRASCO, 2000. p.215.

CANESQUI, A. (Org).

Ciências Sociais e Saúde para o Ensino Médico. São Paulo: Hucitec, 2000.
CASTELLS, M.

A Sociedade em Rede. v. 1, 3. ed. São Paulo: Paz e Terra, 1999.

HAMEL, G;

PRAHALAD, C.K.

Competindo pelo Futuro: Estratégias Inovadoras para Obter o Controle do seu Setor e Criar os Mercados de Amanhã. Rio de Janeiro: Campus, 1995. 


\section{KOTLER, P.}

Administração de Marketing. 2. ed. São Paulo: Atlas, 1991.

MACHADO, M. (Coord.).

Perfil dos Médicos no Brasil. Rio de Janeiro. FIOCRUZ/CFM-MS/PNUD, 1996. Disponível em: <http:// www.ensp.fiocruz.br/perfil $>$. Acesso em 29 dez. 2000.

\section{MAIA, F. J. P.}

Saúde movimenta R\$ 1,8 Bi por ano na Bahia. Gazeta Mercantil, Suplemento Saúde. Disponível em: <http:// www.gazetadabahia.com.br/ saude.html>. Acesso em 2 out. 2000.

PEPPERS, D.;

\section{ROGERS, $M$.}

CRM Series Marketing 1 to $\mathbf{1}$ : Um guia executivo para entender e implantar estratégias de Customer Relationship Management. Jan. 2000. Disponível em: <http:// www.1to1.com.br>. Acesso em 2 out. 2000.
SANT'ANNA, R.N.

O Setor de Serviços na Sociedade da Informação: para a montagem de um sistema de informações e estatísticas. Rio de Janeiro, 1998. Dissertação de Mestrado, PPCI/UFRJ.

\section{SHAPIRO, C.;}

VARIAN, $\mathrm{H}$.

What are the Rules, Anyway? Technology Review, mar/abr., 1999. Disponível em: <http:// www.technologyreview.com/magazine/apr99/print_version/mann.html>. Acesso em: 2 out. 2000.

SVIOKLA, J.;

SHAPIRO, B.

Mantendo Clientes. São Paulo:

Makron Books, 1994.

TAGLIACARNE, G.

Pesquisa de Mercado. São Paulo: Atlas, 1978.

TÉBOUL, J.

A Era dos Serviços. São Paulo: Qualitymark, 1999. 